\title{
Cécilia W. Francis - Robert Viau (dir.), Trajectoires et dérives de la littérature-monde. Poétiques de la relation et $d u$ divers dans les espaces francophones
}

\section{Carminella Biondi}

\section{(2) OpenEdition \\ Journals}

Edizione digitale

URL: http://journals.openedition.org/studifrancesi/2301

DOI: 10.4000/studifrancesi.2301

ISSN: 2421-5856

\section{Editore}

Rosenberg \& Sellier

\section{Edizione cartacea}

Data di pubblicazione: 1 aprile 2014

Paginazione: 191-192

ISSN: 0039-2944

\section{Notizia bibliografica digitale}

Carminella Biondi, « Cécilia W. Francis - Robert Viau (dir.), Trajectoires et dérives de la littérature-monde. Poétiques de la relation et du divers dans les espaces francophones ", Studi Francesi [Online], 172 (LVIII I I) | 2014, online dal 01 avril 2014, consultato il 18 septembre 2020. URL : http://

journals.openedition.org/studifrancesi/2301; DOI : https://doi.org/10.4000/studifrancesi.2301

Questo documento è stato generato automaticamente il 18 settembre 2020.

\section{cc) $($ ) $\ominus$}

Studi Francesi è distribuita con Licenza Creative Commons Attribuzione - Non commerciale - Non opere derivate 4.0 Internazionale. 


\title{
Cécilia W. Francis - Robert Viau (dir.), Trajectoires et dérives de la littérature-monde. Poétiques de la relation et du divers dans les espaces francophones
}

\author{
Carminella Biondi
}

\section{NOTIZIA}

CÉCILIA W. FRANCIS - ROBERT VIAU (dir.), Trajectoires et dérives de la littérature-monde. Poétiques de la relation et du divers dans les espaces francophones, Amsterdam-New York, Rodopi, «Francopolyphonies», pp. 605.

1 Se il numero di pagine in cui si affronta un argomento letterario (o altro) è indice della sua importanza o, quanto meno, della sua attualità, bisogna riconoscere che il progetto lanciato il 16 marzo 2007 in Francia dal manifesto Pour une littérature-monde en français seguito poi, poco dopo, dal volume dello stesso titolo (liberato dal limitativo "en français"), presso l'editore Gallimard a cura di Michel Le Bris e di Jean Rouaud e da Je est un autre. Pour une identité-monde, nel 2010, a cura degli stessi e presso lo stesso editore ha continuato a sollecitare l'attenzione dei critici, sollevando polemiche, ripulse violente, consensi, dibattiti. Molti di questi ultimi sono focalizzati sull'inevitabile rapporto dialettico con il concetto di francofonia, di cui il manifesto aveva decretato il superamento e quindi la morte, ma che continua invece, a sua volta, a far versare fiumi di inchiostro. Le finalità del volume sono indicate dai curatori nella quarta di copertina, che riprende, in sintesi, le idee espresse nell'articolo introduttivo (La littérature-monde: vers une dynamique repensée du centre et de la périphérie): «Par ce livre, nous espérons apporter une contribution pertinente aux échanges théoriques et empiriques entourant 
la "littérature-monde", concept devenu incontournable pour quiconque est soucieux du développement et de l'avenir de la littérature de langue française». Ed è indubbio che il volume rappresenti un apporto "pertinente" al dibattito contribuendo, proprio grazie alla sua ampiezza e alla ricchezza di voci, a individuarne il rayonnement a trecentosessanta gradi (anche se i critici convocati sono per più di un terzo canadesi, come i curatori); ma spesso le voci, come è giusto che sia in ogni dibattito letterario e in particolare attorno a un soggetto tanto difficile da definire e da circoscrivere, sono discordanti, fino a oscillare tra due poli opposti: si tratta di un'operazione di avanguardia o di retroguardia, si sfonda un muro invalicabile o una porta aperta?, portando a una conclusione, se di conclusione si può parlare, quanto mai aperta sulla fruibilità e l'operatività del concetto in ambito critico, anche se la maggioranza degli interventi riconosce che il manifesto ha gettato un sasso che ha smosso le acque (qualcuno parla di "morta gora") della letteratura francese e ha posto - se in maniera adeguata lo si vedrà in seguito - un problema non più eludibile dopo l'avvento delle grandi letterature cosiddette "periferiche", che chiedono un campo letterario policentrico di lingua francese, in cui la Francia non abbia più un ruolo predominante, come sembra aver continuato ad avere all'interno del "sistema francofonia". Anche sull'origine del concetto "littérature-monde", di cui si sottolinea a più riprese l'ambiguità, gli interventi sono discordanti: molti dei critici ne riconoscono l'ascendenza glissantiana (nel bene e nel male), negata invece da uno dei promotori del manifesto, Michel Le Bris: «[La littérature-monde] ne procède pas de la pensée de Glissant. Cette affaire, elle a une origine qui date de la réflexion, je disais, sur le totalitarisme, autour de Sartre, de Foucault, etc.» (p. 579). Forse entrambe le posizioni sono vere perché il percorso sotterraneo, spesso inconscio, del concetto sembra aver lambito molti lidi prima di trovare una formulazione, e uno di questi lidi è indubbiamente la poetica di Glissant, che nel volume qui presentato è oggetto sia di studi diretti che di riferimenti molteplici.

2 È impossibile scendere nello specifico di un volume così ampio e così ricco, in cui ogni singolo intervento porta il suo piccolo o grande contributo al dibattito. Mi soffermerò pertanto, dopo una descrizione generale dei contenuti, sugli articoli più "teorici" della prima parte e sulla tavola rotonda che conclude il volume, anche se i capitoli centrali, in cui il concetto è messo alla prova attraverso l'analisi di "casi", quali un'opera, un'area geografica, un genere, non sono meno stimolanti. Il volume è diviso in otto parti: si apre con la discussione teorica del concetto (anche in rapporto ad altri concetti affini o complementari: francofonia, postcolonialismo, transcultura...), per poi verificarne la tenuta attraverso la lettura critica di alcune letterature o una serie di analisi testuali: I «Généalogie d'un concept: en amont et en aval de la littératuremonde» (pp. 15-129); II «Littérature-monde: lectures et formes du roman» (pp. 133-214); III «Voix transcontinentales et littérature-monde» (pp. 217-299); IV «Paradigmes transfrontaliers et littérature-monde» (pp. 303-374); V «Les relations, le rhizome, le divers» (pp. 377-456); VI «Oralité, diglossie et plurilinguisme» (pp. 459-517); VII «Création littéraire dans le contexte de la littérature-monde» (pp. 521-564); VIII «Points saillants de la table ronde "Quel est l'avenir de la littérature monde?"» (pp. 565-584). Chiudono il volume una breve bio-bibliografia dei trenta collaboratori e una Postface che riproduce il Manifesto del marzo 2007.

3 La prima parte si compone di cinque interventi che, come si è detto, dibattono il concetto di "littérature-monde", cercando di individuarne l'origine, i pregi, i limiti e il 
possibile rayonnement. Apre Lydie MOUDILENo con un saggio (Le manifeste comme écho. Échos du manifeste) che ne ricostruisce i precedenti, il contesto e si interroga sugli sviluppi ancora difficilmente ipotizzabili: «Il reste à savoir, bien sûr, dans quelle mesure la production future, quels que soient ses labels, aura confirmé cette délivrance proclamée et fait résonner cet appel à l'innovation, à la remise en question d'assignations identitaires et au retour de la poésie si emphatiquement annoncé depuis quelques années déjà» (p. 27). Jorge CALDÉRON, nel saggio che segue, precisa fin dal titolo quali sono le finalità del suo intervento: Les implications du manifeste «Pour une "littérature-monde" en français", ma costella il suo testo di molti condizionali, a dimostrazione della difficoltà di cogliere appieno il senso dell'operazione manifestaria dei "44" (è il numero dei firmatari del manifesto Pour une littérature-monde en français) e le sue possibili implicazioni: «L'étude de la littérature-monde d'expression française pourrait par exemple...», «le but principal de l'analyse de la littérature-monde serait d'étudier, de comprendre et d'expliquer la manière dont les œuvres littéraires écrites en français depuis, par exemple, la fin des guerres d'indépendance explorent le rapport entre les nations, l'État-nation, la littérature et l'histoire culturelle» (p. 49). 0 ancora: «Une des caractéristiques principales des œuvres faisant partie de la littérature-monde pourrait être...», «Les œuvres ne s'inscriraient pas directement...» (p. 49), ecc. Régine ROBIN, il cui romanzo La Québécoite (1983) è diventato l'emblema della letteratura migrante, ritraccia il lungo percorso che, attraverso varie ricerche, anche nominalistiche (transculture, interculture, écriture métisse...), ha portato al manifesto Pourune littérature-monde, il quale non fa che prendere atto dei cambiamenti avvenuti: «pour une nouvelle génération d'écrivains, la multiplicité des langues et des cultures va de soi» (p. 67). Anche nel suo intervento alla tavola rotonda, pur insistendo sul "malentendu" che, a suo parere, è alla base del manifesto («ça s'adresse à ce qui ne va pas en France sur le plan d'institutions littéraires, sur le plan de la production littéraire», p. 580) e sul suo carattere spesso prescrittivo (confessa che il «retour du roman de la représentation» le fa cadere le braccia, p. 581), Robin riconosce che ha avuto una funzione dinamizzante molto positiva nell'ambito delle lettere di lingua francese. Il saggio di uno dei curatori, Robert VIAU (La littérature-monde en français: l'historique d'une querelle), ricostruisce gli antecedenti immediati del manifesto e gli aspetti salienti delle reazioni che ha suscitato. È importante per capire come proprio nei due o tre anni, e in particolare nei mesi, che ne hanno preceduto la pubblicazione, nel mondo delle lettere francesi fosse in atto tutto un fiorire di contestazioni contro il "centro" e contro la Francofonia come istituzione (è il saggio che si sofferma maggiormente sui termini del confronto "Littérature-monde"/Francofonia), accompagnato da una ricerca di riposizionamenti nella galassia francofona. Si tratta di uno dei saggi che meglio contribuisce a ricostruire la posta in gioco, i bisogni, ma anche le passioni che sono confluite, quasi una sorta di urlo liberatorio, nel dibattito che ha preceduto e seguito la pubblicazione del manifesto. Il critico conclude il suo lavoro chiedendosi se si tratti veramente di una "révolution copernicienne", come avevano un po' enfaticamente affermato i firmatari, ma è pronto a riconoscere che «Malgré ses détracteurs, il faut bien admettre que E pur si muove» (p. 106). Conclude la prima parte del volume il saggio di Kathleen M. GYSSELS, «Pour une littérature-monde»: Appellation d'Orgine Contrôlée pour une littérature défrancisisée au XxI siècle? che, pur avendo come fulcro la letteratura caraibica, allarga il discorso alla tematica della "littératuremonde" e in particolare al manifesto, che le sembra si sia infilato in un vicolo cieco, chiedendo la debalcanizzazione, ma avendo ancora come fulcro e cible Parigi e la 
Francia (e non è la sola a insistere su questa contraddizione). La Gyssels sponsorizza, come veramente innovatori sul piano dell'apertura e della decentralizzazione, autori da lei definiti "francophiles anglophones" che pubblicano in zone "periferiche", come Nancy Huston e Russel Banks, ritenuti «les voix promettantes d'une littérature illimitée qui, tant thématiquement qu'artistiquement, décloisonne» (p. 125).

4 Conclude il volume, come si è detto, la sintesi di una tavola rotonda tenutasi all'Università Saint-Thoms (New Brunswick) il 23 ottobre 2010, che ha come protagonisti François Paré (moderatore), Alain Borer, Michel Le Bris, Lise Gauvin e Régine Robin. I toni sono spesso anche molto aspri, con Le Bris sulla difensiva che precisa il senso dell'operazione 'manifesto' cercando di chiarire gli aspetti contestati in alcuni interventi, e soprattutto difende il manifesto dall'"accusa" di aver condotto dal centro e per il centro, una battaglia decentratrice, di voler abbattere frontiere e modelli che il carattere "normativo" del manifesto di fatto ricostruisce, ecc. Ma, nonostante le critiche e i distinguo, il giudizio d'insieme sul lancio della «littérature-monde» è positivo e può essere riassunto nelle parole conclusive di Régine Robin: «ça fait du bruit, mais pas au sens que ça nous réveille, ça nous interpelle, ça nous fait discuter, même si on s'engueule, ça permet de lancer des idées nouvelles, je crois que ça c'est très positif» (p. 584).

Risulta evidente che questo imponente volume, in cui confluiscono i risultati delle posizioni critiche più diverse sollevate dal manifesto e dal concetto di "littératuremonde", è un testo di riferimento imprescindibile non solo per chi si interessi alla tematica specifica, ma per tutti coloro che si interrogano sul legame fra opera letteraria $\mathrm{e}$ comunità di appartenenza $\mathrm{e}$, più in generale, sul senso e la funzione della letteratura nel mondo di oggi. 